\title{
Kidney aging process and the management of the elderly patient with renal impairment (Review)
}

\author{
RALUCA IOANA PAPACOCEA ${ }^{1 *}$, DELIA TIMOFTE ${ }^{2}$, MARIA-DANIELA TANASESCU $^{3,4}$, \\ ANDRA-ELENA BALCANGIU-STROESCU ${ }^{2,5^{*}}$, DANIELA GABRIELA BALAN ${ }^{5}$, \\ ADRIAN TULIN $^{6,7}$, OVIDIU STIRU ${ }^{8,9}$, ILEANA ADELA VACAROIU ${ }^{10,11}$, ANDRADA MIHAI ${ }^{12,13}$, \\ CRISTIAN CONSTANTIN POPA ${ }^{14,15}$, CRISTINA-ILEANA COSCONEL ${ }^{16}$, MIHALY ENYEDI ${ }^{6,17,}$ \\ DANIELA MIRICESCU ${ }^{18}$, LAURA RADUCU ${ }^{19,20^{*}}$ and DORIN IONESCU ${ }^{2,3^{*}}$
}

${ }^{1}$ Discipline of Physiology, Faculty of Medicine, 'Carol Davila' University of Medicine and Pharmacy, 020021 Bucharest;

${ }^{2}$ Department of Dialysis, Emergency University Hospital, 050098 Bucharest; ${ }^{3}$ Department of Medical Semiology,

Discipline of Internal Medicine I and Nephrology, Faculty of Medicine, 'Carol Davila' University of Medicine and Pharmacy, 020021 Bucharest; ${ }^{4}$ Department of Nephrology, Emergency University Hospital, 050098 Bucharest;

${ }^{5}$ Discipline of Physiology, Faculty of Dental Medicine, 'Carol Davila' University of Medicine and Pharmacy;

${ }^{6}$ Department of Anatomy, Faculty of Medicine, 'Carol Davila' University of Medicine and Pharmacy, 020021 Bucharest;

${ }^{7}$ Department of General Surgery, 'Prof. Dr. Agrippa Ionescu' Clinical Emergency Hospital, 011356 Bucharest;

${ }^{8}$ Department of Cardiovascular Surgery, Faculty of Medicine, 'Carol Davila' University of Medicine and Pharmacy,

020021 Bucharest; ${ }^{9}$ Department of Cardiovascular Surgery, 'Prof. Dr. C.C. Iliescu' Emergency Institute for Cardiovascular Diseases, 022322 Bucharest; ${ }^{10}$ Department of Nephrology and Dialysis, St. John Emergency Clinical Hospital, 042122 Bucharest; ${ }^{11}$ Department of Nephrology, Faculty of Medicine, 'Carol Davila' University of Medicine and Pharmacy;

${ }^{12}$ Discipline of Diabetes, Nutrition and Metabolic Diseases-N. Paulescu National Institute, Faculty of Medicine,

'Carol Davila' University of Medicine and Pharmacy, 020021 Bucharest; ${ }^{13}$ Department II of Diabetes,

Prof. N. Paulescu, Bucharest, Nutrition and Metabolic Diseases National Institute of Diabetes, Nutrition and

Metabolic Disease, 020474 Bucharest; ${ }^{14}$ Department of Surgery, Faculty of Medicine, 'Carol Davila' University of Medicine and Pharmacy, 020021 Bucharest; ${ }^{15}$ Department of Surgery, Emergency University Hospital, 050098 Bucharest;

${ }^{16}$ Discipline of Foreign Languages, Faculty of Dental Medicine, 'Carol Davila' University of Medicine and Pharmacy,

020021 Bucharest; ${ }^{17}$ Department of Radiology, 'Victor Babes' Private Medical Clinic, 030303 Bucharest;

${ }^{18}$ Discipline of Biochemistry, Faculty of Dental Medicine, 'Carol Davila' University of Medicine and Pharmacy;

${ }^{19}$ Discipline of Plastic and Reconstructive Surgery, Faculty of Medicine, 'Carol Davila' University of Medicine and Pharmacy, 020021 Bucharest; ${ }^{20}$ Department of Plastic and Reconstructive Surgery 'Prof. Dr. Agrippa Ionescu'

Clinical Emergency Hospital, 011356 Bucharest, Romania

Received October 16, 2020; Accepted November 17, 2020

DOI: $10.3892 /$ etm.2021.9697

\begin{abstract}
As life expectancy increases, a rise in the number of chronically ill patients is observed due to the aging population.

Correspondence to: Dr Andra-Elena Balcangiu-Stroescu, Department of Dialysis, Emergency University Hospital, Spaiul Independenţei 169, 050098 Bucharest, Romania

E-mail: stroescu_andra@yahoo.ro

*Contributed equally
\end{abstract}

Key words: aging, elderly patients, renal aging, chronic kidney disease, morbidity
Among the various diseases, chronic kidney disease is at present one of the main causes of morbidity and, due to its typical complications, it is also one of the most important causes of mortality in the general population. For these reasons, the understanding of the kidney aging process, its consequences and its adequate management are essential. The judicious use of certain types of drugs, the prevention of episodes of renal injury either by toxic mechanisms or by dehydration are important aspects and are part of the apropriate approach for elderly patients. The most effective treatment of various types of conditions with a negative impact on renal function and for which an increased incidence is known as we age should also be considered. Thus, in the case of elderly patients, in order to protect the kidneys, an integrative approach is recommended, one that includes both elements of prevention and the appropriate treatment of existing diseases. 


\section{Contents}

1. Introduction

2. Renal aging

3. Renal aging-structural changes

4. Renal aging-physiological changes

5. Renal aging-biomarkers

6. Renal aging-clinical significance and patient management

7. Conclusions

\section{Introduction}

The increase in life expectancy has led to the need to prevent early senescence, by promoting healthy aging and maintaining the quality of life for as long as possible. In other words, the aim is to maintain the ability of the body to function properly until old age, through pharmacological and non-pharmacological methods. The concept of functional ability is accepted by the World Health Organisation and is part of the definition of healthy aging $(1,2)$. The aging process is found in all organs. A large number of studies deal with the kidney involvement within this process (3). Thus, along with aging, comes the development of a specific phenotype (as far as the kidney is concerned) which encapsulates the decrease of the renal parenchyma mass (predominantly at the cortical level), increased vascular resistance and decreased renal perfusion (4). The outcome of these changes is represented by the onset of glomerulosclerosis and interstitial fibrosis, tubular atrophy and last but not least, of arteriosclerosis (4). Hydroelectrolytic and metabolic disorders occur in elderly patients secondary to the aforementioned changes (3).

The kidney is subjected to numerous structural changes which will later cause the disruption of the physiological processes. Thus, the varying number of nephrons (between 700.000 and 1.8 million) decreases with age (5). According to the literature studies, approximately 6,000 nephrons/kidney are lost annually (6). The number of nephrons is directly proportional to the weight of a person at birth, thus, in patients with a smaller number of nephrons the process is quickened (5). The early onset of hypertension and chronic kidney disease also contribute to the process (5).

Chronic kidney disease (CKD) represents one of the clinical models of aging (7).

The main risk factors leading to CKD are high blood pressure and diabetes $(8,9)$. CKD is also associated with a series of other factors, such as: sex, race, family history, low birth weight, obesity, socio-economic status, smoking, various systemic diseases, and genetic, toxic or obstructive factors (10-14).

The increased kidney damage in male patients is due to hormone influence, according to studies (13-17). The androgen hormones are also considered a factor in the progression of the CKD (16).

Female sex hormones, acting through the receptors at this level, have a protective role in the kidney aging process. Estrogens prevent fibrosis by influencing certain processes specific to the aging process, such as decrease of the collagen synthesis. On the other hand, male sex hormones have an important profibrotic action. Testosterone stimulates matrix expansion, and also plays a key role in accelerating the apoptosis of the convoluted proximal tubule cells. It is, thus, easy to understand the fact that in women, in whom diminished estrogen and increased testosterone levels occur during the aging process, the risk of developing kidney degenerative lesions is higher $(18,19)$. One of the characteristics of kidney aging is glomerular sclerosis, markedly more important in male patients. The stimulation of the production of the extracellular matrix by the androgen hormones as well as the increase of the metalloproteinases in female patients are the factors responsible for the glomerular sclerosis related to the sex of the patient. There is also a sex-related difference in the renal plasma flow. In fact, a smaller plasma flow is described in females compared to males. The early diminished glomerular filtration rate (GFR) can also be observed in women $(18,19)$. An important factor in the differentiation between sexes is played by the beneficial influence of estrogen on nitric oxide synthetase (NOS). A later increase of one of the NOS inhibitors-asymmetric dimethylarginine (ADMA) is found in female patients $(13,18,19)$. In opposition, it should be mentioned that androgen hormones stimulate the renin-angiotensin-aldosterone system (RAAS). This in turn leads to the increase of blood pressure and amplifies the kidney degenerative changes $(18,19)$.

The association of obesity with clinical conditions including endothelial dysfunction, chronic inflammation, prothrombotic state and hypervolemia plays a significant role in the onset of CKD. Another association is smoking, which represents a key risk factor for CKD (14). Chronic kidney disease can also be caused by systemic diseases, such as autoimmune diseases, cardiovascular diseases, hepatic diseases, viral infections (VHB, VHC and HIV) and certain neoplasms (20).

The episodes of acute kidney injury (AKI) represent risk factors for CKD, as well. This is mainly due to inadequate recovery factors including the expression of the kidney injury molecule-1 (KIM-1), the presence of chronic inflammatory infiltrate and microvascular changes, recurrent tubule injury, profibrotic factor secretion, interstitial collagen and glomerulosclerosis deposition. These are all mechanisms responsible for the kidney aging related post-acute injuries (21).

\section{Renal aging}

Literature studies emphasize the important role of the Klotho protein in preventing the aging process $(13,22,23)$. In kidneys, this protein is mainly localized in the distal convoluted tubule $(13,23)$. Klotho protein acts as a co-receptor for the fibroblast growth factor 23 (FGF-23) and thus, the activation of FGF receptor by FGF-23 depends on Klotho presence. FGF-23 is a phosphaturic hormone produced in the bones. Its main activity, regulation of the mineral-bone metabolism, is performed through the following mechanisms: The maintenance of the serum phosphorus levels within normal limits, the stimulation of the parathyroid hormone synthesis (PTH) and vitamin D3 secretion in kidney $(22,23)$.

The influence of Klotho in kidney aging prevention can be inferred from its physiologic roles. Klotho reduces oxidative stress, regulates phosphocalcic metabolism, and endothelium-depedendent vascular reactivity, as well as modulates the activity of the calcium channels in the tubular 
cells. The increase of oxidative stress, the chronic stimulation of angiotensin II (AT-II) and CKD decrease Klotho activity (13). A reduced Klotho level and an increased FGF-23 serum level accelerate the aging in CKD and the onset of arteriosclerosis (22). Muscle and skin atrophy, osteoporosis and ectopic calcifications were identified in mice with Klotho deficiency (22). Concurrently, literature studies have described the enhancement of vascular changes and the interstitial fibrosis along with Klotho transfection, emphasizing the importance of the protein in kidney degeneration (13). The decrease of the expression level of Klotho in the aging process has been demonstrated to cause not only the increase of FGF-23 (a factor with a major impact on cardiovascular morbidity and mortality) serum level, but also the activation of the Wnt signaling pathway. During the aging process, the decrease of the FGF-23 and the activation of the Wnt signaling pathway represent the indirect way through which Klotho reduction influences the onset of the kidney fibrosis (22).

Medical progress has led to the development of therapeutic agents which, among other roles, have the ability to increase Klotho expression. Some of these agents are: peroxisome proliferator-activated receptor gamma (PPAR- $\gamma$ ) activators, angiotensin receptor blockers (BRA), statins and active D vitamin derivatives (23). Other benefits are mentioned for some of these drugs in connection to the kidney aging prevention. Secondary to the administration of PPAR- $\gamma$ agonists, Klotho expression increases and oxidative stress and kidney inflammation decrease. Taking into account the increase of AT II with aging and the augmentation of the oxidative stress and kidney fibrosis, the benefit of BRA in the improvement of degenerative kidney changes can be assessed (23).

The changes in telomere length contribute to abrupt kidney aging. These nucleotide sequences are formed in the presence of telomerase and are found at the end of the chromosomes. Their role is to protect the genetic material and to block chromosome degradation. The enzyme related to formation of telomeres cannot be identified in the kidney, which explains why telomere shortening is frequently found in the kidney during the aging process. The shortening of telomeres leads to aging and cellular apoptosis through cell instability. It should also be mentioned that the shortening of the telomeres is one of the factors that prevent ischemic postinjury recovery $(13,23)$.

Oxidative stress appears as a result of the imbalance between the production and the degradation of free radicals and generates tissue injuries. Both vascular and non-vascular enzymes contribute to the development of oxygen reactive species, emphasizing the importantance of nicotinamide adenine dinucleotide phosphate (NADPH) oxidase, dysfunctional mitochondrial respiratory chain, lipoxygenase and xanthine oxidase in the onset of oxidative stress (24). A large segment of the degenerative changes of the old kidney occur secondary to important oxidative stress. According to findings, aging reduces renal perfusion and increases ADMA synthesis, with an important role in the shortening of the telomeres. The increased level of homocysteine, which is a constant presence in patients with CKD, has an indirect effect of NOS suppression (25).

In patients with CKD there is a series of elements with a substantial contribution to the increase of oxidative stress, including: aging, chronic iron therapy, and the presence of comorbidities (such as HBP, diabetes and dyslipidemia). The increase of oxidative stress has been revealed to be correlated with the decrease of the glomerular filtration rate $(24,26)$. In diabetic patients oxidative stress is incriminated in the development of microvascular complications (27).

The decrease of antioxidant capacity is identified in patients with CKD, along with the other mechanisms that increase oxidative stress that were aforementioned. Studies conducted on animals have demonstrated the decrease of glutathione reductase (GSH) and $\mathrm{Cu} / \mathrm{Zn}$-SOD catalase (22). Patients with CKD also present with a low level of vitamin C, as a result of a diet poor in fruit and vegetables (prevention of hyperpotassemia) (24).

The sirtuin family is another class of molecules with an antioxidant effect. Its decrease with aging increases oxidative stress in patients with CKD (22).

The disruption of cell growth, accompanied by cell resistance to apoptosis, along with the inability of the immune system to eliminate them, are the main features of the cellular senescence (7).

Aged cells have a specific phenotype-senescenceassociated secretory phenotype (SASP), characterized by the release of proinflammatory cytokines which create a chronic inflammatory status which influence tissue functions (7).

Cellular aging is also a trigger for fibrosis, and some of the factors contributing to its onset are: Interleukin-6 (IL-6), interleukin-8 (IL-8), growth-regulated oncogene-alpha (GRO alpha) and Wnt16B (22).

According to studies, the medulla is the area with the largest number of senescent cells. The explanation for this phenomenon is the hypo-oxygenation of the area and the increased level of the oxidative stress (22).

The deposition of abnormal collagen (type I and III) is responsible for the development of fibrosis and takes place by attracting a large number of leukocytes, associated with the action of matrix metallopeptidase 1 (MMP1) inhibitor to support the change of MMPs to fibrosis (22).

\section{Renal aging-structural changes}

Microscopic evaluation of the aged kidneys has revealed the most significant alterations including: Nephrosclerosis (glomerulosclerosis, interstitial fibrosis, tubular atrophy and arteriosclerosis) and nephron hypertrophy (5). Glomerulosclerosis and tubular atrophy are consequences of the ischemic injury triggered by arteriosclerosis. These changes are completed with the deposition of hyaline material on the Bowman capsule, causing the collapse of the glomeruli and their global sclerosis, which is a common occurrence in elderly patients. A GFR decrease is also found in these patients. It is worth mentioning the fact that no causal relationship has been established between the two entities, which are independent.

Glomerular hypertrophy is frequently associated with diabetes and obesity. This change is also commonly found in kidney diseases or after surgery causing a reduction of the kidney mass. One reason for this change could be the association between global glomerular sclerosis and the hypertrophy of the remaining functional nephrons (5). Notable is the fact that nephrosclerosis is closely associated with the age of patients, while glomerular hypertrophy is associated with 
obesity, hyperuricemia and less with old age (5). It should also be mentioned that nephrosclerosis is associated with high blood pressure, but that there is no association between this and body mass index (BMI), albumin urinary excretion or the level of serum cholesterol and uric acid (22). The thickening of the glomerular membrane and mesangial expansion are found in elderly patients as well as the nephrosclerosis and glomerular hypertrophy (13).

Literature describes changes in kidney volume as the patient grows older. The accompanying glomerulosclerosis and tubular atrophy are an explanation for the reduction in cortical kidney volume (5).

A decrease in urinary tubules similar to the reduction in the number of glomeruli is described in the aging process. The association between tubular atrophy, with the presence of tubular diverticula, the thickening of tubular basement membrane and interstitial fibrosis are commonly encountered (13).

A large number of vascular alterations are found in elderly patients. Features to be noticed are the hypertrophy of the media and intima, with increased prevalence of arteriosclerosis and atherosclerosis. Vascular lesions are triggered by increased cell proliferation, the increase of the extracellular matrix and the presence of capillary shunts, especially at the cortical level (22).

\section{Renal aging-physiological changes}

The structural changes occurring in the aged kidney cause the disruption of renal physiology. Glomerular hyperperfusion, which is initially an adaptive mechanism, will eventually lead to glomerular hypertension (13). During the aging process, a decrease of glomerular filtration rate (GFR) is described but this is not correlated with the degree of glomerulosclerosis which depends on age and is not secondary to kidney impairment as part of cardiovascular diseases, according to studies (13). Concurrently, another study describes the correlation between the decrease of GFR and the vascular involvement, as well as the poor protein diet of elderly patients. The importance of periodic GFR evaluation can be understood from the fact that it is an important prognostic element for the development of cardiovascular events and the mortality of patients (5).

The disturbance in the physiologic processes at the tubule level, secondary to structural changes there, leads to the development of a phenotype with the following features: The inability to balance the normal sodium concentration, predisposing to dehydration (disturbs conservation), or to HBP (disturbs elimination), a tendency to the development of hyperpotassemia, the alteration of tubule functions of concentrating and diluting urine and the tendency to metabolic acidosis $(13,22)$.

The structural changes of the vessels trigger a series of physiologic alterations. Thus, the renal plasma flow diminishes with aging, especially in the kidney cortex. The association of the decreased renal plasma flow with the increase of the post-glomerular resistance is meant to maintain GFR within normal limits for a period (13). Vasoconstriction is supported by the increase of sympathetic tone and the alteration of vasodilator substances (i.e., NO) (22).
Endocrine changes are observed in elderly patients, secondary to kidney alteration during aging. The release of the renal eicosanoids (prostaglandins, prostacyclins, thromboxane, leukotrienes), the activation of the RAA system as well as the secretion of the erythropoietin and vitamin $\mathrm{D}$ is also changed in these patients. With old age and the development of renal dysfunction, most patients have a certain degree of anemia. This anemia is caused by the reduction of erythropoietin synthesis (EPO), characteristic in elderly patients with kidney impairment. The level of serum vitamin $\mathrm{D}$ is reduced in these patients, as well. According to present data, the kidney incapacity to hydrolyze 25 hydroxyvitamin $\mathrm{D}$ in position 1 and the low sun exposure of patients contribute to low serum levels of vitamin D (13).

\section{Renal aging-biomarkers}

The predisposition of elderly patients to develop AKI is without exception accepted in studies. In the presence of degenerative kidney lesions, the development of an acute injury leads to a deterioration of the prognosis in these patients. In the early stages of the disease, the clinical picture of kidney damage following acute renal injury is absent (GFR unchanged, with no alterations in diuresis); this is why it is necessary to use an instrument able to identify the subclinical lesions and to allow immediate therapeutic intervention when necessary. In order to solve this problem, the researchers suggested the use of specific biomarkers to identify early kidney lesions secondary to acute injury, such as: KIM-1, L-FABP, lipocalin (NGAL), cystatin C, IL-18, TIMP-2 and IGFBP7 (28).

Neutrophil gelatinase-associated lipocalin (NGAL), is a biomarker that has been demonstrated to be efficient in detecting AKI occurring secondary to ischemia or nephrotoxic substances (29). Notably, NGAL discriminates between pre-renal AKI and acute tubule interstitial nephropathy (ATN). The same discrimination can also be made by calprotectin; this biomarker is not specific to kidney impairment, since high levels can be found in other pathologies, such as intestinal inflammatory diseases, rheumatoid arthritis, and prostate cancer.

KIM-1 is another biomarker that can detect the presence of ATN. It can be measured in urine in early post-injury stages, and its high level is related to the lethal outcome of patients (30).

L-FABP, due to the fact that it is induced by kidney ischemia, has been suggested as a marker of hypoxia.

Cystatin $\mathrm{C}$ has demontrated better efficacy than the estimated glomerular filtration rate (eGFR) formula in identifying the decrease of kidney function and glomerular filtration (30).

The evidence regarding the ability of TIMP-2 x IGFBP-7 to predict the risk of developing AKI, the need for hemodialysis or the risk of death, render these biomarkers useful to the clinician in identifying the patients at risk of developing complications and in selecting those that must undergo specific preventative measures and treatment. Moreover, to evaluate the probability of developing AKI in the following $12 \mathrm{~h}$, the FDA approved, in 2014, the use of a test called NephroCheck, which includes these two biomarkers (30). The possible explanation for the use of these two biomarkers is that certain studies describe the fact that cellular stress stimulates 
their synthesis. More recent studies argue that the presence of these biomarkers in urine is secondary to their glomerular hyperfiltration (TIMP-2, IGFBP-7 extrarenal production) and to their reduced reabsorption due to tubular damage (29).

Another molecule with a role in suppressing the cell cycle is p21. This plays an important role in the suppression of the cell cycle at the tubule level. The disruption of normal the cell cycle has a certain influence on the kidney aging process. Similar to TIMP-2, IGFBP-7, secondary to extrarenal production, p21 level is increased in patients with AKI. These two observations emphasize the usefulness of the $\mathrm{p} 21$ biomarker in the early detection of systemic and renal degenerative changes (32).

\section{Renal aging-clinical significance and patient management}

From a clinical point of view, the structural and functional changes in the kidneys during the aging process require the special attention of the clinician. Elderly patients are at risk for dehydration and AKI secondary to it, or for hyperpotassemia, with negative cardiovascular consequences $(13,22,33)$. In order to avoid AKIs, with the possible deterioration of pre-existing lesions, nonsteroidal anti-inflammatory drugs and contrast substances must be administered with special precautions to elderly patients (5). Since the aging process is accompanied by a decrease in the glomerular filtration rate and a decrease in the elimination of hydrosoluble drugs, it is necessary to administer them judiciously with diminished doses depending on the degree of kidney impairment (34).

After the age of 40 , there is a physiologic decrease in the glomerular filtration rate (32). According to study findings, the mere presence of decreased glomerular filtration is not a criterion per se to diagnose CKD (35). Thus, in elderly patients, it is recommended that the diagnosis and the stage of the CKD should encompass GFR evaluation based on the equation of the glomerular filtration rate and the evaluation of the presence of other urinary changes, such as albuminuria. In fact, studies have not demonstrated an association between increased negative events and the decrease of glomerular filtration rate alone (34). It is known that CKD increases the cardiovascular risk of patients. The assessment of the urinary loss of albumin is necessary in order to stratify cardiovascular risk in elderly patients with varying degrees of kidney alteration. In numerous studies, albuminuria is closely associated with cardiovascular diseases and is a recognized marker $(34,36)$. Recent studies also describe the Cystatin $\mathrm{C}$ effectiveness in the evaluation of the cardiovascular involvement (34). The presence of cardiovascular comorbidities is also an important factor in the increase of mortality in patients with chronic kidney disease $(37,38)$.

High blood pressure is a clinical condition identified in a significant number of elderly patients (39) from which a considerable percentage have CKD. Systolic blood pressure is generally higher in this group of patients (40). A large proportion of patients suffering from CKD also have mineral and bone disorders and vascular calcifications which, by increasing the arterial stiffness, trigger HBP. These vascular changes are more intense in the chronic hemodialyzed patients $(41,42)$. A high level of uric acid is associated with the increase of blood pressure and it is an important risk factor for cardiovascular diseases (43).

The management of HBP in elderly patients with CKD requires treatment. Taking into account the strong antiproteinuric effect of angiotensin-converting-enzyme inhibitors (ACEI) and of the angiotensin receptor blockers, these classes of drugs are the first intention therapies in elderly CKD patients. The blood pressure target is individually established for each patient in spite of the general recommendations in the present guides, which suggest maintaining the blood pressure under $130 / 80 \mathrm{mmHg}$ in this group of patients; episodes of orthostatic hypotension followed by the supplementary decrease of GFR decrease should also be avoided. Patient monitorization allows for the identification of the increase of creatinine secondary to the treatment and the necessity of treatment cessation (44). In chronic hemodialysis patients, the use of hypotensive therapy of one of the two classes is recommended, with the addition of either beta-blockers, or blockers of calcium channels when necessary (45).

It must be mentioned that age plays an important contribution in the progression of the chronic kidney disease toward end stages. In extremely old patients, the development of kidney impairment from early to late stages is highly unlikely if only the aging process is taken into account. The decrease of the GFR during several years, as well as the fact that the patient can sucumb are part of the explanation (34). Nevertheless, in some elderly patients, CKD may progress to end stages. The identification of these patients, their careful monitorization as well as the apropriate management are key elements to improving survival (36).

The evolution of the CKD to advanced stages, which require extrarenal epuration methods, makes it necessary for the clinician to consider all aspects related to the geriatric patient. Thus, the decision to start renal replacement therapy (RRT) in a geriatric patient requires the analysis of several aspects, such as: the choice of treatment (hemodialysis, peritoneal dialysis, transplant), the unquestionable benefit of the procedure on long- or short-term prognosis, the possibility of vascular status approach, the compliance to treatment (dementia, comorbidities) of the patient, the quality of life of the patient. For some of the aspects considered before taking the decision to initiate the extrarenal epuration therapy, the scales tilt towards postponing it and maintaining the conservative treatment. On the other hand, when the survival of the elderly patient requiring RRT is taken into account, irrespective of the therapeutic method selected, it is shorter than the survival of younger patients, but better than that of elderly patients with conservative treatment (36).

\section{Conclusions}

Aging is a physiologic process of life. Like skin aging, which is easily identifiable by merely looking at the patient, body aging is an unavoidable process, characterized by the development of lesions with clinical consequences for the kidney. The careful management of the elderly patient with kidney impairment certainly brings benefits to this group of patients, preventing the deterioration of renal injuries, and facilitating the proper approach during later stages of the disease. 


\section{Acknowledgements}

Not applicable.

\section{Funding}

No funding was received.

\section{Availability of data and materials}

All data generated or analyzed during this study are included in this published article.

\section{Authors' contributions}

RIP, DT, MDT, AEBS, DGB, AT, OS, IAV, AM, PCC, CIC, ME, DM, LR and DI designed the study and wrote the manuscript and performed a literature search and selected the included studies. RIP, DT, MDT, AEBS, DGB, AT, OS, IAV, AM, PCC, CIC, ME, DM, LR and DI critically revised the manuscript. All authors read and approved the final manuscript. The contributions of all the authors on this review are greatly valued and appreciated.

\section{Ethics approval and consent to participate}

Not applicable.

\section{Patient consent for publication}

Not applicable.

\section{Competing interests}

The authors declare that they have no competing interests.

\section{References}

1. Kalache A, de Hoogh AI, Howlett SE, Kennedy B, Eggerdorfer M, Marsman DS, Shao A and Griffiths JC: Nutrition interventions for healthy ageing across the lifespan: A conference report. Eur J Nutr 58 (Suppl 1): S1-S11, 2019.

2. https://www.who.int/ageing/healthy-ageing/en/. Accessed April 25, 2020.

3. Alvis BD and Hughes CG: Physiology considerations in geriatric patients. Anesthesiol Clin 33: 447-456, 2015.

4. Yang HC and Fogo AB: Fibrosis and renal aging. Kidney Int Suppl (2011) 4: 75-78, 2014

5. Denic A, Glassock RJ and Rule AD: Structural and functional changes with the aging kidney. Adv Chronic Kidney Dis 23: 19-28, 2016.

6. Hommos MS, Glassock RJ and Rule AD: Structural and functional changes in human kidneys with healthy aging. J Am Soc Nephrol 28: 2838-2844, 2017.

7. Dai L, Qureshi AR, Witasp A, Lindholm B and Stenvinkel P: Early vascular ageing and cellular senescence in chronic kidney disease. Comput Struct Biotechnol J 17: 721-729, 2019.

8. Diaconu C: Treatment of diabetes in patients with heart failure. Proceedings of the 3rd international conference on interdisciplinary management of diabetes mellitus and its complications-diabetes mellitus in internal medicine, interdiab 2017, Serafinceanu C, Negoiţă O and Elian V (eds), pp170-177, 2017.

9. Dobrică EC, Gaman MA, Cozma MA, Bratu OG, Pantea Stoian A, and Diaconu CC: Polypharmacy in type 2 diabetes mellitus: Insights from an internal medicine department. Medicina (Kaunas) 55: 436, 2019.
10. Tanasescu MD, Balcangiu Stroescu AE, Raducu L, Limbau AM, Diaconescu AC, Balan DG and Ionescu D: Uterine cervical cancer and chronic kidney disease an association that should not be skipped. Rev Chim 69: 2524-2526, 2018.

11. Balan DG, Balcangiu-Stroescu AE, Tanasescu MD, Diaconescu A, Raducu L, Mihai A, Tanase M, Stanescu II and Ionescu D: Nutritional intervention in patients with diabetic renal disease-a brief presentation. Rev Chim 69: 4078-4082, 2018.

12. Balcangiu-Stroescu AE, Tanasescu MD, Diaconescu A, Raducu L, Balan DG, Mihai A, Tanase M, Stanescu II and Ionescu D: Diabetic nephropathy: A concise assessment of the causes, risk factors and implications in diabetic patients. Rev Chim 69: 3118-3121, 2018.

13. Bolignano D, Mattace-Raso F, Sijbrands EJ and Zoccali C: The aging kidney revisited: A systematic review. Ageing Res Rev 14: 65-80, 2014.

14. Kazancioğlu R: Risk factors for chronic kidney disease: An update. Kidney Int Suppl (2011) 3: 368-371, 2013.

15. Chang PY, Chien LN, Lin YF, Wu MS, Chiu WT and Chiou HY: Risk factors of gender for renal progression in patients with early chronic kidney disease. Medicine (Baltimore) 95: e4203, 2016.

16. Duru OK, Li S, Jurkovitz C, Bakris G, Brown W, Chen SC, Collins A, Klag M, McCullough PA, McGill J, et al: Race and sex differences in hypertension control in CKD: Results from the kidney early evaluation program (KEEP). Am J Kidney Dis 51: 192-198, 2008.

17. Reckelhoff JF, Zhang $\mathrm{H}$ and Granger JP: Decline in renal hemodynamic function in aging SHR: Role of androgens. Hypertension 30: 677-681, 1997.

18. Weinstein JR and Anderson S: The aging kidney: Physiological changes. Adv Chronic Kidney Dis 17: 302-307, 2010.

19. Gava AL, Freitas FP, Meyrelles SS, Silva IV and Graceli JB: Gender-dependent effects of aging on the kidney. Braz J Med Biol Res 44: 905-913, 2011.

20. Rajashekar A, Perazella MA and Crowley S: Systemic diseases with renal manifestations. Prim Care 35: 297-328, 2008.

21. Ferenbach DA and Bonventre JV: Mechanisms of maladaptive repair after AKI leading to accelerated kidney ageing and CKD. Nat Rev Nephrol 11: 264-276, 2015.

22. O'Sullivan ED, Hughes J and Ferenbach DA: Renal aging: Causes and consequences. J Am Soc Nephrol 28: 407-420, 2017.

23. Lu X and Hu MC: Klotho/FGF23 axis in chronic kidney disease and cardiovascular disease. Kidney Dis (Basel) 3: 15-23, 2017.

24. Kao MP, Ang DS, Pall A and Struthers AD: Oxidative stress in renal dysfunction: Mechanisms, clinical sequelae and therapeutic options. J Hum Hypertens 24: 1-8, 2010.

25. Liguori I, Russo G, Curcio F, Bulli G, Aran L, Della-Morte D, Gargiulo T, Cacciatore F, Bonaduce D and Abete P: Oxidative stress, aging, and diseases. Clin Interv Aging 13: 757-772, 2018.

26. Cachofeiro V, Goicochea M, de Vinuesa SG, Oubiña P, Lahera V and Luño J: Oxidative stress and inflammation, a link between chronic kidney disease and cardiovascular disease. Kidney Int Suppl: S4-S9, 2008.

27. Totan A, Balcangiu-Stroescu AE, Melescanu Imre M, Miricescu D, Balan DG, Stanescu II, Ionescu D, Timofte D, Tanasescu MD and Greabu M: XOR-possible correlations with oxidative stress and inflammation markers in the context of diabetic kidney disease. Rev Chim 70: 1396-1398, 2019.

28. Bonventre JV: Current biomarkers in kidney disease: Dawning of a new era. https://www.kidneynews.org/kidney-news/ special-sections/kidney-disease-biomarkers/current-biomarkers-inkidney-disease-dawning-of-new-era. Accessed April 25, 2020.

29. Ortega LM and Heung M: The use of cell cycle arrest biomarkers in the early detection of acute kidney injury. Is this the new renal troponin? Nefrologia 38: 361-367, 2018 (In English, Spanish).

30. Sakhuja A: Novel biomarkers of renal function introduction and overview. https://emedicine. medscape.com/ article/1925619-overview\#a3. Accessed April 25, 2020.

31. Johnson ACM and Zager RA: Mechanisms underlying increased TIMP2 and IGFBP7 urinary excretion in experimental AKI. J Am Soc Nephrol 29: 2157-2167, 2018.

32. Johnson AC and Zager RA: Plasma and urinary p21: Potential biomarkers of AKI and renal aging. Am J Physiol Renal Physiol 315: F1329-F1335, 2018.

33. Kovesdy CP: Updates in hyperkalemia: Outcomes and therapeutic strategies. Rev Endocr Metab Disord 18: 41-47, 2017.

34. Rule AD and Glassock RJ: The aging kidney. https://www.uptodate.com/contents/the-aging-kidney. Accessed May 5,2020. 
35. Fassett RG: Current and emerging treatment options for the elderly patient with chronic kidney disease. Clin Interv Aging 9: 191-199, 2014.

36. Prakash S and O'Hare AM: Interaction of aging and chronic kidney disease. Semin Nephrol 29: 497-503, 2009.

37. Balcangiu-Stroescu AE, Tanasescu MD, Diaconescu A, Raducu L, Constantin AM, Balan DG, Tarmure V and Ionescu D: Cardiovascular comorbidities, inflammation and serum albumin levels in a group of hemodialysis patients. Rev Chim 69: 926-929, 2018.

38. Gaman MA, Dobrica EC, Pascu EG, Cozma MA, Epingeac ME, Gaman AM, Pantea Stoian A, Bratu OG and Diaconu CC: Cardio metabolic risk factors for atrial fibrillation in type 2 diabetes mellitus: Focus on hypertension, metabolic syndrome and obesity. J Mind Med Sci 6: 157-161, 2019.

39. Egan BM: Treatment of hypertension in older adults, particularly isolated systolic hypertension. https://www.uptodate.com/ contents/treatment-of-hypertension-in-older-adults-particularlyisolated-systolic-hypertension. Accessed May 5,2020

40. Cai G, Zheng Y, Sun X and Chen X; Survey of Prevalence, Awareness, and Treatment Rates in Chronic Kidney Disease Patients with Hypertension in China Collaborative Group: Prevalence, awareness, treatment, and control of hypertension in elderly adults with chronic kidney disease: Results from the survey of prevalence, awareness, and treatment rates in chronic kidney disease patients with hypertension in China. J Am Geriatr Soc 61: 2160-2167, 2013.
41. Timofte D, Ionescu D, Medrihan L, Mandita A, Rasina A and Damian L: Vascular calcification and bone disease in hemodialysis patients assessment, association and risk factors. Nephrol Dial Transplant 22: 325-326, 2007.

42. Timofte D, Dragos D, Balcangiu-Stroescu AE, Tănăsescu MD, Gabriela Bălan D, Răducu L, Tulin A, Stiru O and Ionescu D: Abdominal aortic calcification in predialysis patients: Contribution of traditional and uremia-related risk factors. Exp Ther Med 20: 97-102, 2020.

43. Timofte D, Mandita A, Balcangiu-Stroescu AE, Balan DG, Raducu L, Tanasescu MD, Diaconescu AC, Dorin D, Cosconel CI and Ionescu D: Hyperuricemia and cardiovascular diseases-clinical and paraclinical correlations. Rev Chim 70: 1045-1046, 2019.

44. Abad K, Rivera FX and Owen JG: The Management of hypertension in elderly patients with chronic kidney disease. J Clin Outcomes Manag 25: 211-218, 2018.

45. Mandita A, Timofte D, Balcangiu-Stroescu AE, Balan D, Raducu L, Tanasescu MD, Diaconescu A, Dragos D, Cosconel CI, Stoicescu SM and Ionescu D: Treatment of high blood pressure in patients with chronic renal disease. Rev Chim (Bucharest) 70: 993-995, 2019.

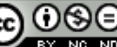

This work is licensed under a Creative Commons Attribution-NonCommercial-NoDerivatives 4.0 International (CC BY-NC-ND 4.0) License. 